\title{
EUMETSAT Copernicus Marine Training and User Support
}

Hayley Evers-King, (Hayley.EversKing@eumetsat.int, @HayleyEversKing)

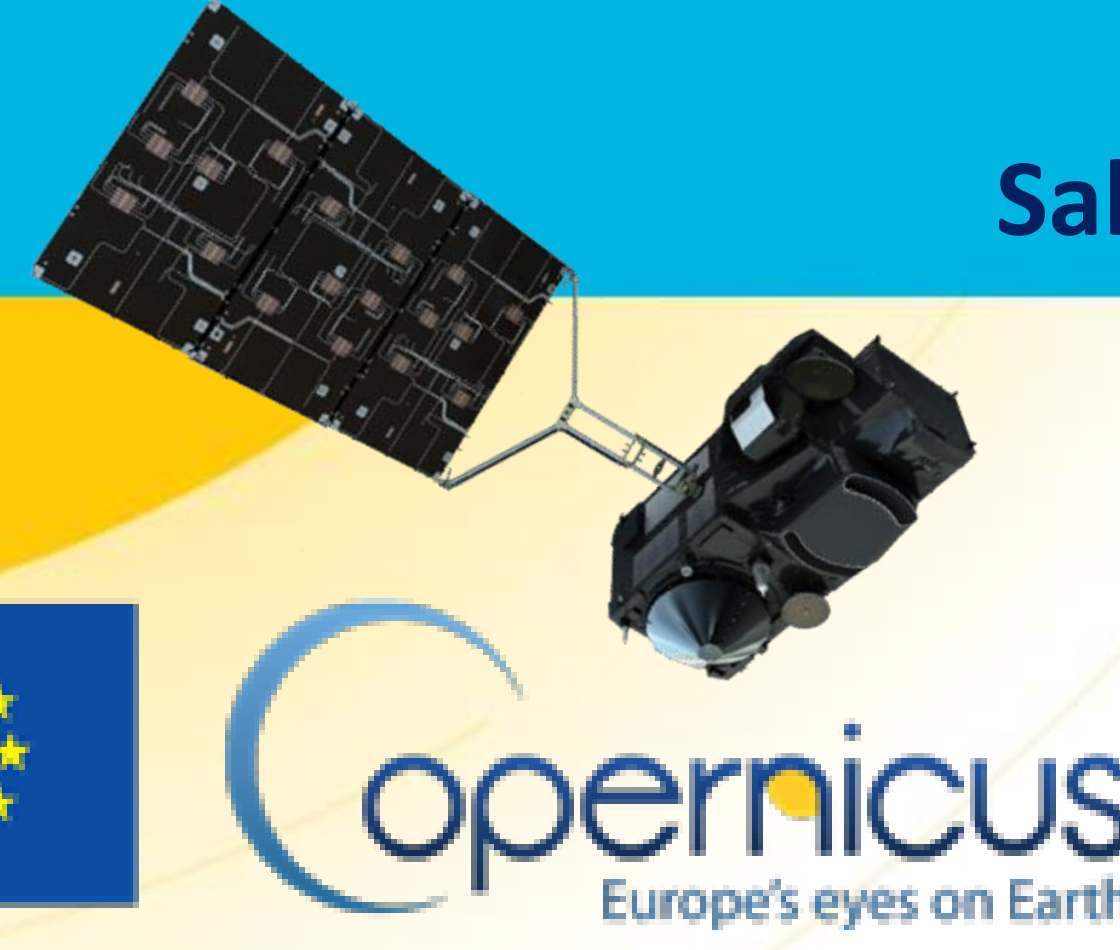
Stream (CMDS). The CMDS refers to all the level 1 and level 2 marine data from sensors on the Sentinel-3, Jason-3, and upcoming Sentinel-6 satellites, including ocean colour, sea surface temperature, and surface topography data.

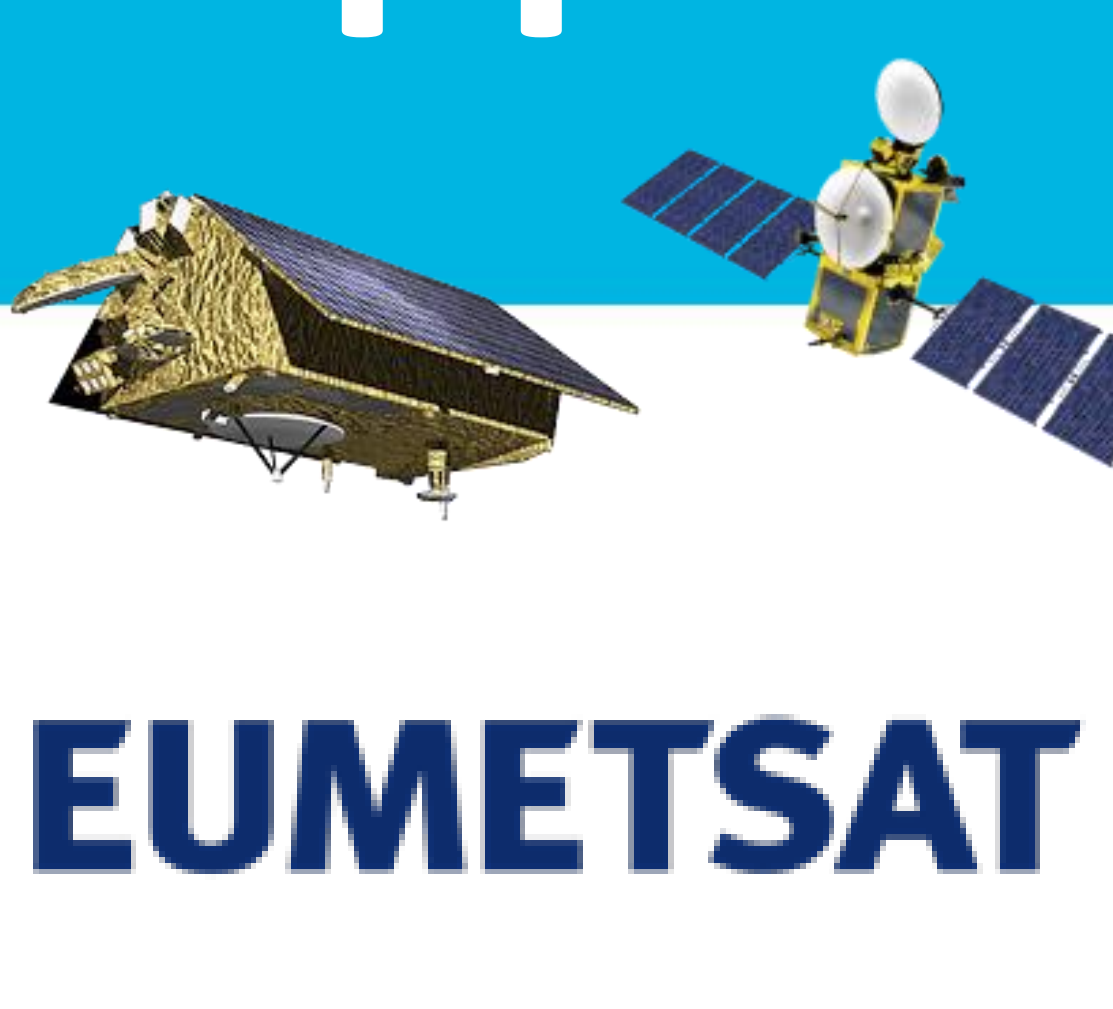

\section{Data access}

EUMETSAT provides access to data from the Copernicus missions it operates through a variety of methods to suit users needs. This includes push services, web based portals, APIs and hosted processing. New data services, in the pilot phase, are coming soon!
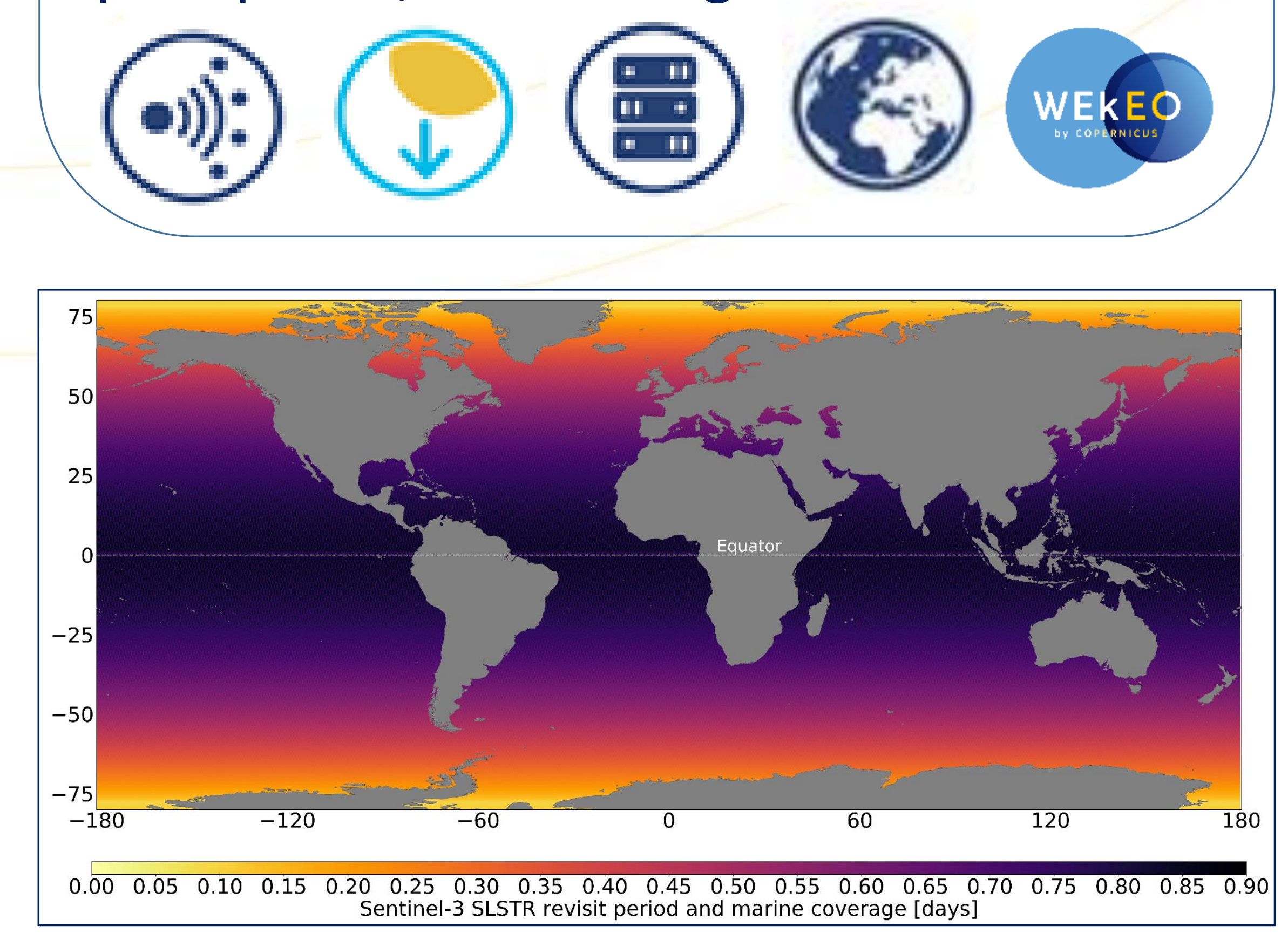

\section{Contact us!}

- For questions about our data and services: ops@eumetsat.int

- For questions about, or to collaborate on training: copernicus.training@eumetsat.int

- Twitter: @eumetsat \& @eumetsat users

\section{User support and engagement} EUMETSAT provides a helpdesk for user queries via ops@eumetsat.int. Product information is available through handbooks and ATBDs, along with further details on reprocessing campaigns at http://slstr.eumetsat.int. We also feature case studies of interesting images, and user studies and applications on our website.
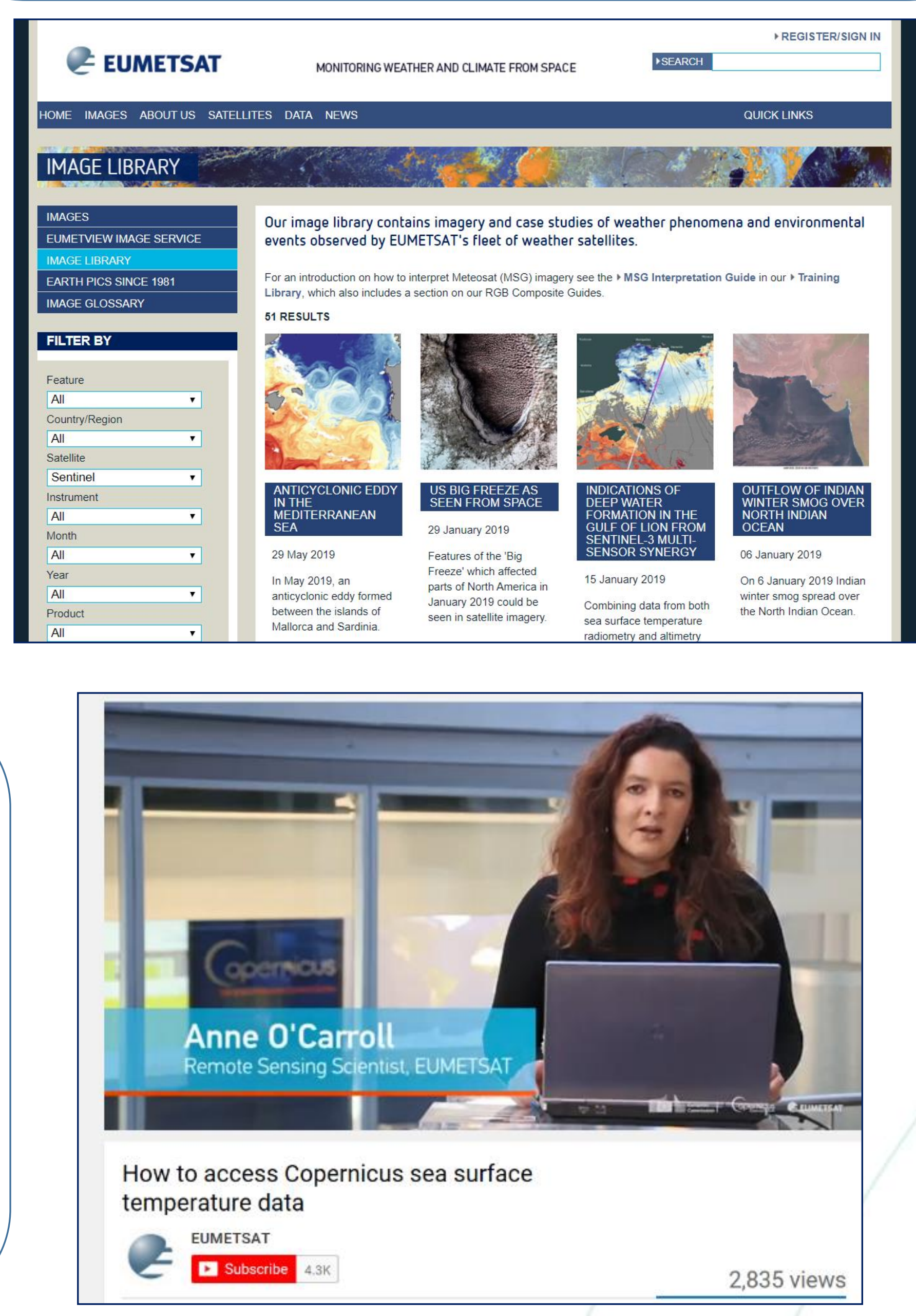

\section{Training}

We run training courses online, in-person, and collaboratively (providing experts to support community initiatives). Our Massive Open Online Courses including "Monitoring the oceans from space" are online and free to re-use, along with our YouTube videos. Our training includes Python scripts and Jupyter notebook tools and tutorials available through GitLab. We can support conferences and courses with short term mobility support funding. Further information and upcoming events can be found on our training website.

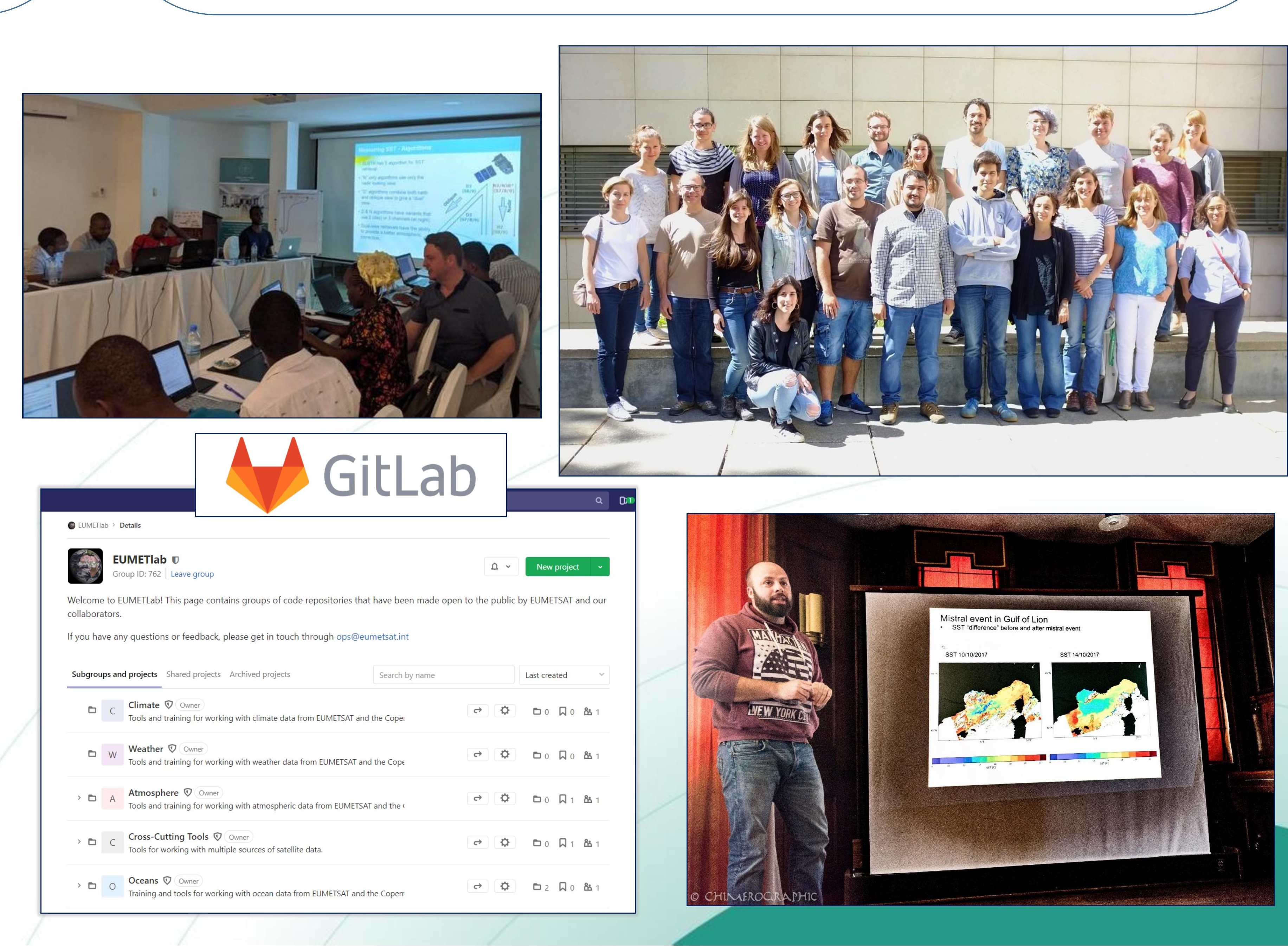

